\title{
Global sensitivity analysis of backside coating parameters on dynamic response of AM-AFM
}

\author{
Kaveh E. Torkanpouri*, Hassan Zohoor**, Moharam H. Korayem*** \\ *Department of Mechanical and Aerospace Engineering, Science and Research Branch, Islamic Azad University, Tehran \\ Iran \\ **Center of Excellence in Design, Robotics and Automation (CEDRA), Mechanical Engineering Department, Sharif \\ University of Technology, Tehran, Iran \\ The Academy of Sciences of IR Iran, Tehran, Iran, E-mail: zohoor@sharif.edu \\ ***Department of Mechanical and Aerospace Engineering, Science and Research Branch, Islamic Azad University, \\ Tehran, Iran \\ Robotic Research Laboratory, Center of Excellence in Experimental Solid Mechanics and Dynamics, School of \\ Mechanical Engineering, Iran University of Science and Technology, Tehran, Iran.
}

cross $^{\text {ref }}$ http://dx.doi.org/10.5755/j01.mech.23.2.13908

\section{Introduction}

The Atomic force microscope (AFM) [1] is widely used in engineering science and has found many applications in different sciences. AFM use a micro cantilever as a probe for scanning the sample surface which is externally oscillated close to its resonance frequency in the dynamic modes [2]. The excitations are mostly with harmonically base excitation [3]. Amplitude, phase and resonance frequency are modified by tip-sample interaction forces $[2,3]$. These changes in oscillation with respect to the external reference oscillation provide information about the sample's characteristics [4]. Dynamic AFM methods are generally divided to Amplitude Modulated Atomic Force Microscope (AM-AFM) [3] and Frequency Modulated Atomic Force Microscope (FM-AFM) [5]. In AM-AFM, ratio of value of amplitude in scanning with free amplitude is the source of contrast. The base excitation amplitude is as large as $20 \mathrm{~nm}$ to $100 \mathrm{~nm}$ [2]. This large amount of excitation amplitude cause working the AM-AFM in both the attractive and repulsive regime and so this method names sometimes the Tapping mode [6]. The detection system that has become the standard method for AM-AFM used a laser that is reflected from the backside of the cantilever onto detector. The signal forms the four quadrants of the detector are compared to calculate the deflection signal [7]. Reflectivity or reflectance of cantilever is very important in the quality of received signals by photodiode. In the normal incident the refractive index of silicon nitride with wavelength of 0.3136 $\mu \mathrm{m}$ is 2.148 and the reflectivity of silicon nitride can be calculated $13 \%$. This means just $13 \%$ of the incident power is reflected [7]. To improve signal from the cantilevers, they are often sputter coated with a film of various metals in the backside of cantilever [7, 8]. Even a thin coating of a metal can dramatically change the dynamic behaviour of AFM cantilever $[9,10]$. By adding a layer of metal coating, the natural resonant frequency of the cantilever will vary [11]. Various coatings are used depending of applications $[8,9$, $12,13]$. Different methods of calibration have been proposed where the effect of backside coating is omitted [3, 1416], however some attempts were done by assuming the coating as an inelastic body for calibration of AFM cantilever [7].
A hybrid method for calibration of AFM cantilevers was introduced where the effects of backside coating is included. The method was based on the minimization of the difference between the Finite Element Method (FEM) and experimentally determined full-field displacement maps of the surface of the cantilever in motion at several resonant frequencies in far away from the sample [17]. When the AFM is far away of the sample the effects of interaction force is negligible and it will not affect dynamic response of system. A systematic investigation of AFM cantilevers coated with several thicknesses of the Diamond like carbon films is studied experimentally in faraway condition. It was demonstrated that for coated cantilevers, the resonance frequency will be determined not only by the properties of the cantilever, but also by the elastic modulus and thickness of the coating [9]. Cantilevers of AFM that uniformly coated with metal thin films between 18 and $73 \mathrm{~nm}$ were studied experimentally. An approximate equation for calculating coated AFM cantilever based on Euler Bernoulli theory is introduced while the AFM is faraway of the sample. It was remarked the effect of coating is significant in natural frequency of cantilever [10].

Global sensitivity analysis (GSA) aims at investigating the influence of variations in input parameters on the variation of a model outputs. It can identify contributions of different inputs to the in full distribution domain of the inputs and comprehensively consider the average effect of the inputs on the outputs. GSA can also provide the importance sequence of the model inputs. One type of GSA is the variance-based Sobol' method [18]. In Variance-based method the sensitivity of the output to an input variable is measured by the amount of variance in the output that is caused by that input. Such methods decompose the output variance into parts attributable to individual input variables as well as their interactions. This method has been applied on various models.

For AFM cantilevers, different parameters are studied recently for nano-manipulation $[19,20]$ piezoelectric micro-cantilever [21], Carbon Nanotube Characteristics in tapping mode [22] and for liquid environments [23]. But in all studies, the interactions between parameters are omitted and just the first order indices discussed. 
In current study effects of backside coating parameters including, elastic modulus, thickness, specific mass and Poisson ratio are investigated in AM-AFM. For this approach, Timoshenko beam theory with concentrated tip mass for coated cantilever is derived and the mode shapes of AM-AFM in faraway situation are calculated. The effect of interaction force is modelled by nonlinear general model [24] and assume mode method [25] is used for determination of time response of equations of motion. GSA based on Sobol's statistical method $[18,26]$ are applied to identify and prioritize the most influential inputs and identify noninfluential coating parameters and their interactions on dynamic response of AM-AFM in faraway and scanning situation.

\section{Mathematical model}

The schematic configuration of AM-AFM is figured in Fig. 1. The extended Hamilton's principle is expressed.

$$
\int_{t_{1}}^{t_{2}}\left(\delta T-\delta U+\delta W^{n c}\right) d t=0
$$

where $T, U$ and $W^{n c}$ are kinetic energy, strain energy and work by tip-sample interaction force of the system respectively.

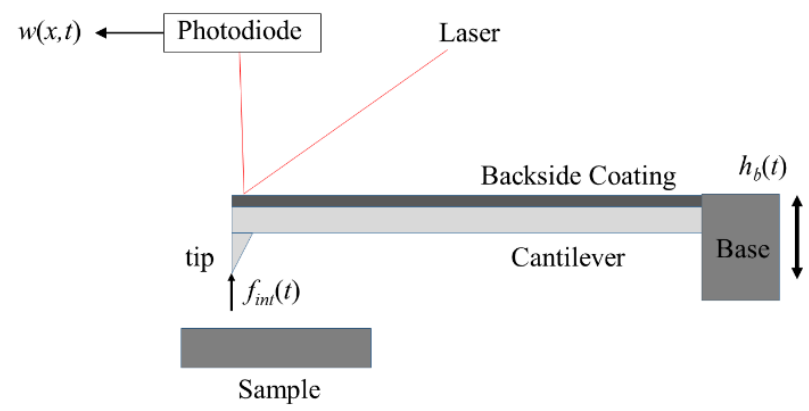

Fig. 1 Schematic of AM-AFM cantilever with backside coating

$$
\begin{aligned}
& T=\frac{1}{2} \int_{0}^{L} \rho_{i} A_{i}\left[\dot{h}_{b}(t)+\dot{w}(x, t)\right]^{2} d x+ \\
& +\frac{1}{2} m_{t i p}\left[\dot{h}_{b}(t)+\dot{w}(L, t)\right]^{2}+\frac{1}{2} \int_{0}^{L} \rho_{i} I_{i}[\dot{\theta}(x, t)]^{2} d x ;(2) \\
& U=\frac{1}{2} \int_{0}^{L} E_{i} I_{i}\left[\theta^{\prime}(x, t)\right]^{2} d x+ \\
& +\frac{1}{2} \int_{0}^{L} k_{i} A_{i} G_{i}\left[w^{\prime}(x, t)-\theta(x, t)\right]^{2} d x,
\end{aligned}
$$

where $i$ is a dummy index and $i=1$ and 2 are related to substrate/cantilever and coating respectively in the mathematical expression. $w(x, t)$ is relative deflection of cantilever with respect to base and $\theta(x, t)$ is the slope of cantilever that is defined in Timoshenko beam theory. "Prime" and "dot" present the partial derivative with respect to spatial coordinate and time respectively. $m_{\text {tip }}$ is the tip mass of cantilever, $f_{\text {int }}(t)$ is nonlinear nano-scale tip-sample interaction force and $h_{b}(t)$ is the base excitation.

The base excitation is assumed a harmonic motion with amplitude equals to $Y_{0}$ and frequency equal to resonance frequency of AM-AFM in faraway situation. Other parameters are introduced in Table 1 . The variation of work and energy terms are determined. Integrating over time using integration by parts theorem results the required terms of Eq. (1) as following:

$$
\begin{aligned}
& \int_{t_{1}}^{t_{2}} \delta T \cdot d t=-\int_{t_{1}}^{t_{2}} \int_{0}^{L} \rho_{i} I_{i} \ddot{\theta}(x, t) \delta \theta(x, t) \cdot d x d t- \\
& -\int_{t_{1}}^{t_{2}} m_{t i p}\left[\ddot{h_{b}}(t)+\ddot{w}(L, t)\right] \delta w(L, t) d t- \\
& -\int_{t_{1}}^{t_{2}} \int_{0}^{L} \rho_{i} A_{i}\left[\ddot{h_{b}}(t)+\ddot{w}(x, t)\right] \delta w(x, t) d x d t ; \\
& \int_{t_{1}}^{t_{2}} \delta U d t=-\left.\int_{t_{1}}^{t_{2}} E_{i} I_{i} \theta^{\prime}(x, t) \delta \theta(x, t)\right|_{0} ^{L} d t+ \\
& +\int_{t_{1}}^{t_{2}} \int_{0}^{L} E_{i} I_{i} \theta^{\prime \prime}(x, t) \delta \theta(x, t) d x d t- \\
& -\int_{t_{1}}^{t_{2}} k_{i} A_{i} G_{i}\left\{\left.\left[w^{\prime}(x, t)-\theta(x, t)\right] \delta w(x, t)\right|_{0} ^{L}\right\} d t+ \\
& +\int_{t_{1}}^{t_{2}} \int_{0}^{L} k_{i} A_{i} G_{i}\left[w^{\prime \prime}(x, t)-\theta(x, t)\right] \delta w(x, t) d x d t+ \\
& +\int_{t_{1}}^{t_{2}} \int_{0}^{L} k_{i} A_{i} G_{i}\left[w^{\prime}(x, t)-\theta(x, t)\right] \delta \theta(x, t) d t d x ; \\
& \delta W^{n c}=f_{i n}(t) \delta w(L, t)- \\
& -C \int_{0}^{L} \dot{w}^{\prime}(x, t) \delta w(L, t) d x .
\end{aligned}
$$

Introducing Eqs. (4)-(5) in to Eq. (1) and using the calculus of variation the two coupled partial equations of motion for Timoshenko beam theory with concentrated end mass [27] for backside coated cantilever are determined:

$$
\begin{aligned}
& \rho_{i} A_{i}\left[\ddot{h_{b}}(t)+\ddot{w}(x, t)\right]+C \dot{w}^{\prime}(x, t)- \\
& -k_{i} A_{i} G_{i}\left[w^{\prime \prime}(x, t)-\theta^{\prime}(x, t)\right]=0 ; \\
& -\rho_{i} I_{i} \ddot{\theta}(x, t)+E_{i} I_{i} \theta^{\prime \prime}(x, t)+ \\
& +k_{i} A_{i} G_{i}\left[w^{\prime}(x, t)-\theta(x, t)\right]=0 .
\end{aligned}
$$

For the case of clamped-free beam the final form of boundary conditions using Eqs. (4)-(6) are presented as:

$$
\begin{aligned}
& \theta(0, t)=0, \quad \theta^{\prime}(L, t)=0, w(0, t)=0 ; \\
& -m_{t i p}\left[\ddot{h}_{b}(t)+\ddot{w}(L, t)\right]- \\
& -k_{i} A_{i} G_{i}\left[w^{\prime}(L, t)-\theta(L, t)\right]+f_{\text {int }}(t)=0 .
\end{aligned}
$$

Eq. (10) shows that, the boundary conditions of motion are non-homogeneous, therefore a new variable $Z(x, t)$ is introduced for homogenizing $[3,5]$.

$$
w(x, t)=Z(x, t)+f_{\text {int }}(t) g(x) .
$$

where the function $g(x)$ is chose such the multiplier of $f_{\text {int }}(t)$ and $\ddot{f}_{\text {int }}(t)$ be eliminated in Eq. (10). Then the function $g(x)$ should satisfy Eqs. (12).

$$
g(0)=0, g(\mathrm{~L})=0, g^{\prime}(\mathrm{L})=\frac{1}{k_{i} A_{i} G_{i}} .
$$

The simplest guess for $g(x)$ is a quadratic function as following which satisfies Eqs. (12). 


$$
g(x)=\frac{-1}{k_{i} A_{i} G_{i}} x+\frac{1}{k_{i} A_{i} G_{i} L} x^{2} .
$$

Eq. (11) is introduced to higher order PDE which is resulted from the combination of Eqs. (7) and (8). After some calculation the equation of motion is presented as following:

$$
\begin{aligned}
& -\frac{\left(\rho_{i} A_{i}\right)\left(E_{i} I_{i}\right)}{\left(k_{i} A_{i} G_{i}\right)\left(\rho_{i} I_{i}\right)} \ddot{w}^{\prime \prime}(x, t)+\frac{E_{i} I_{i}}{\rho_{i} I_{i}} w^{\prime \prime \prime}(x, t)+ \\
& +\frac{\rho_{i} A_{i}}{\rho_{i} I_{i}}\left[\ddot{h_{b}}(t)+\ddot{w}(x, t)\right]+\frac{C}{\rho_{i} I_{i}} \dot{w}^{\prime}(x, t)- \\
& -\frac{C .\left(E_{i} I_{i}\right)}{\left(k_{i} A_{i} G_{i}\right)\left(\rho_{i} I_{i}\right)} \dot{w}^{\prime \prime \prime}(x, t)-\ddot{w}^{\prime \prime}(x, t)+ \\
& +\frac{\rho_{i} A_{i}}{k_{i} A_{i} G_{i}}\left[\dddot{h_{b}}(t)+\dddot{w}(x, t)\right]+\frac{C}{k_{i} A_{i} G_{i}} \dddot{w}^{\prime}(x, t)=0 .
\end{aligned}
$$

Assumed mode method [25] is used on $\mathrm{Z}(x, t)$. Here $\varphi_{i}(x)$ are mode functions of AM-AFM in faraway condition and $q_{i}(t)$ are generalized coordinates.

$$
Z(x, t)=\sum_{k=1}^{N} \varphi_{k}(x) q_{k}(t) .
$$

The mode shapes of monolayer undamped Timoshenko beam with concentrated end mass has been solved analytically [28]. The same process are applied to coated cantilever. After some mathematical calculation, the characteristic equation is determined as:

$$
\begin{aligned}
& \left(\frac{\alpha^{2}+s^{2}}{\alpha}\right)\left(R_{3} R_{4}^{\prime}-R_{3}^{\prime} R_{4}+R_{4} R_{1}^{\prime}-R_{1} R_{4}^{\prime}\right)+ \\
& +\left(\frac{\beta^{2}-s^{2}}{\beta}\right)\left(R_{2} R_{3}^{\prime}-R_{2}^{\prime} R_{3}+R_{1} R_{2}^{\prime}-R_{2} R_{1}^{\prime}\right)=0,
\end{aligned}
$$

where

$$
\begin{aligned}
& {\left[\begin{array}{l}
\alpha \\
\beta
\end{array}\right]=\frac{\sqrt{2}}{2}\left\{\mp\left(r^{2}+s^{2}\right)+\left[\left(r^{2}-s^{2}\right)^{2}+\frac{4}{b^{2}}\right]^{1 / 2}\right\}^{1 / 2} ;} \\
& R_{1}=(b / \alpha) \sinh (b \alpha)+M_{n} b^{2} \cosh (b \alpha) ; \\
& R_{2}=(b / \alpha) \cosh (b \alpha)+M_{n} b^{2} \sinh (b \alpha) \text {; } \\
& R_{3}=(b / \beta) \sin (b \beta)+M_{n} b^{2} \cos (b \beta) ; \\
& R_{4}=-(b / \beta) \cos (b \beta)+M_{n} b^{2} \sin (b \beta) ; \\
& R_{1}^{\prime}=\left(\frac{\alpha^{2}+s^{2}}{\alpha}\right) b \alpha \cosh (b \alpha) ; \\
& R_{2}^{\prime}=\left(\frac{\alpha^{2}+s^{2}}{\alpha}\right) b \alpha \sinh (b \alpha) ; \\
& R_{3}^{\prime}=-\left(\frac{\beta^{2}-s^{2}}{\beta}\right) b \beta \cos (b \beta) ; \\
& R_{4}^{\prime}=-\left(\frac{\beta^{2}-s^{2}}{\beta}\right) b \beta \sin (b \beta) .
\end{aligned}
$$

$$
\left.\begin{array}{l}
\xi=\frac{x}{L} ; b=\frac{\rho_{i} A_{i}}{E_{i} I_{i}} L^{4} \omega^{2} ; r^{2}=\frac{\rho_{i} I_{i}}{\rho_{i} A_{i} L^{2}} ; \\
s^{2}=\frac{E_{i} I_{i}}{k_{i} A_{i} G_{i} L^{2}} ; M_{n}=\frac{M}{\rho_{i} A_{i} L} .
\end{array}\right\}
$$

The mode shapes of coated AM-AFM in faraway situation is determined as:

$$
\begin{aligned}
& \varphi(\xi)=\Phi\left[\cosh (b \alpha \xi)-\frac{\left(R_{1}-R_{3}\right)}{\left(R_{2}-R R_{4}\right)} \sinh (b \alpha \xi)-\right. \\
& \left.-\cos (b \beta \xi)+\frac{R\left(R_{1}-R_{3}\right)}{\left(R_{2}-R R_{4}\right)} \sin (b \beta \xi)\right],
\end{aligned}
$$

where $\Phi$ is a constant and:

$$
R=\frac{\left(\alpha^{2}+s^{2}\right)}{\alpha} \frac{\beta}{\left(\beta^{2}-s^{2}\right)} .
$$

Introducing Eq. (11) and (15) to Eq. (14) using the orthogonality of mode functions and integrating over length of cantilever the final form of ODEs are determined as following after some mathematical calculations:

$$
\begin{aligned}
& G_{j} \dddot{q}_{k}+D_{k j} \dddot{q}_{k}+\left(C_{k j}+I_{j}\right) \ddot{q}_{k}+ \\
& \left(B_{k j}+E_{k j}\right) \dot{q}_{k}+A_{k j} q_{k}+F_{j} \dddot{h}_{b}+ \\
& H_{j} \ddot{b}_{b}+O_{j} \dddot{f}_{\text {int }}(t)+M_{j} \dddot{f}_{\text {int }}(t)+ \\
& +\left(L_{j}+P_{j}\right) \ddot{f}_{\text {int }}(t)+\left(K_{j}+N_{j}\right) \dot{f}_{\text {int }}(t)+ \\
& +J_{j} f_{\text {int }}(t)=0,
\end{aligned}
$$

where $j$ is free indices and $k$ is dummy indices which means summation over $k$ and other parameters are noted in Eqs. (24)-(25).

$$
\begin{aligned}
& A_{k j}=\frac{E_{i} I_{i}}{\rho_{i} I_{i}} \int_{0}^{L} \varphi_{k}^{\prime \prime \prime \prime} \varphi_{j} d x ; \\
& B_{k j}=-\frac{C\left(E_{i} I_{i}\right)}{\left(k_{i} A_{i} G_{i}\right)\left(\rho_{i} I_{i}\right)} \int_{0}^{L} \varphi_{k}^{\prime \prime \prime} \varphi_{j} d x ; \\
& C_{k j}=-\left[\frac{\left(\rho_{i} A_{i}\right)\left(E_{i} I_{i}\right)}{\left(k_{i} A_{i} G_{i}\right)\left(\rho_{i} I_{i}\right)}+1\right] \times \int_{0}^{L} \varphi_{k}^{\prime \prime} \varphi_{j} d x ; \\
& D_{k j}=\frac{C}{k_{i} A_{i} G_{i}} \int_{0}^{L} \varphi_{k}^{\prime} \varphi_{j} d x ; \\
& E_{k j}=\frac{C}{\rho_{i} I_{i}} \int_{0}^{L} \varphi_{k}^{\prime} \varphi_{j} d x ; \\
& F_{j}=\frac{\rho_{i} A_{i}}{k_{i} A_{i} G_{i}} \int_{0}^{L} \varphi_{k} d x ; \\
& G_{j}=\frac{\rho_{i} A_{i}}{k_{i} A_{i} G_{i}} \int_{0}^{L} \varphi^{2}{ }_{j} d x ;
\end{aligned}
$$

The non-dimensional variables are defined as: 


$$
\begin{aligned}
& H_{j}=\frac{\rho_{i} A_{i}}{\rho_{i} I_{i}} \int_{0}^{L} \varphi_{j} d x ; \\
& I_{j}=\frac{\rho_{i} A_{i}}{\rho_{i} I_{i}} \int_{0}^{L} \varphi^{2}{ }_{j} d x ; \\
& J_{j}=\frac{E_{i} I_{i}}{\rho_{i} I_{i}} \int_{0}^{L} g^{\prime \prime \prime}(x) \varphi_{j} d x ; \\
& K_{j}=-\frac{C\left(E_{i} I_{i}\right)}{\left(k_{i} A_{i} G_{i}\right)\left(\rho_{i} I_{i}\right)} \int_{0}^{L} g^{\prime \prime \prime}(x) \varphi_{j} d x ; \\
& L_{j}=-\left[\frac{\left(\rho_{i} A_{i}\right)\left(E_{i} I_{i}\right)}{\left(k_{i} A_{i} G_{i}\right)\left(\rho_{i} I_{i}\right)}+1\right] \times \\
& M_{j}=\frac{C}{k_{i} A_{i} G_{i}} \int_{0}^{L} g^{\prime}(x) \varphi_{j} d x ; \\
& N_{j}=\frac{C}{\rho_{i} I_{i}} \int_{0}^{L} g^{\prime}(x) \varphi_{j} d x ; \\
& O_{j}=\frac{\rho_{i} A_{i}}{k_{i} A_{i} G_{i}} \int_{0}^{L} g(x) \varphi_{j} d x ; \\
& P_{j}=\frac{\rho_{i} A_{i}}{\rho_{i} I_{i}} \int_{0}^{L} g(x) \varphi_{j} d x .
\end{aligned}
$$

The nonlinear nanoscale tip-sample interaction force is modeled via general force [24]:

$$
f_{i n}(t)=a_{a}[D(t)]^{\gamma_{a}}+a_{r}[D(t)]^{\gamma_{r}},
$$

where $a_{a}, a_{r}, \gamma_{a}$ and $\gamma_{r}$ denote the attractive and repulsive amplitude and power terms, respectively. $D(t)$ is tip-sample distance and is related to $w(x, t)$ as:

$$
D(t)=w_{\text {set }}+h_{b}(t)+w(L, t),
$$

where $w_{\text {set }}$ is set/approach point of AM-AFM probe. Eq. (27) is reduced to Eq. (28) at $x=L$.

$$
D(t)=w_{s e t}+h_{b}(t)+Z(L, t) .
$$

The cross sectional area of AM-AFM cantilever is usually assumed as rectangular shape [3, 7, 14] however in practice most AFM cantilevers deviate from the rectangular cross section and they mostly have a trapezoidal cross section $[8,17]$ which is due to dynamic etching effect on rectangular cantilevers.

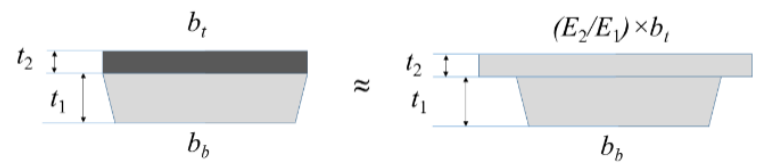

Fig. 2 Transformed cross section of trapezoidal cross section of AM-AFM cantilever

In current research trapezoidal cross section is assumed with $b_{b}$ and $b_{t}$ for bottom wide and top wide of trapezoidal cross section respectively which are showed in Fig. 2. It should be remarked that in all equations $I$ is calculated for transform cross section of bilayer cantilever that is showed in Fig. 2. The details of calculation of transformed cross section can be find in text books and are not noted here.

\section{GSA by Sobol Method}

The mathematical model of AM-AFM is nonlinear and when the model is nonlinear and non-monotonic, the decomposition of the output variance is still defined and can be used [29]. The model input vector is denoted in Eq. (29) for $Y$ as output of system. It is assumed $Y=f(\boldsymbol{\Psi})$ is a square integrable function. It is possible to represent this function as a sum of elementary functions [18].

$$
\begin{gathered}
Y=f(\Psi)=f_{0}+\sum_{i=1}^{d} f_{i}\left(\Psi_{i}\right)+\sum_{i<j}^{d} f_{i j}\left(\Psi_{i}, \Psi_{j}\right)+ \\
+\ldots+f_{123 \ldots d}(\Psi) ; \\
\Psi=\left(\Psi_{1}, \Psi_{2}, \ldots, \Psi_{d}\right) \in R^{d} .
\end{gathered}
$$

This expansion is unique under conditions:

$$
\begin{aligned}
& \int_{0}^{1} f_{i_{1} i_{2} \ldots i_{s}}\left(\Psi_{i_{1}}, \Psi_{i_{2}}, \ldots, \Psi_{i_{s}}\right) d \Psi_{i_{k}}=0, \\
& 1 \leq k \leq s,\left\{i_{1}, \ldots, i_{s}\right\} \subseteq\{1,2, \ldots, d\} .
\end{aligned}
$$

This implies that $f_{0}$ is a constant. It is showed a functional decomposition of the variance is available for output that often is referred to as functional ANalysis Of VAriances (ANOVA).

$$
\begin{gathered}
\operatorname{Var}(Y)=f_{0}+\sum_{i=1}^{d} D_{i}(Y)+\sum_{i<j}^{d} D_{i j}(Y)+\ldots+D_{123 \ldots d}(Y) ; \\
D_{i}(Y)=\operatorname{Var}\left[\mathbb{E}\left(Y \mid \Psi_{i}\right)\right] ; \\
D_{i j}(Y)=\operatorname{Var}\left[\mathbb{E}\left(Y \mid \Psi_{i}, \Psi_{j}\right)\right]-D_{i}(Y)-D_{j}(Y),
\end{gathered}
$$

where $\mathbb{E}$ denotes excepted value that is defined in mathematical symbols.

For higher order interactions, the similar mathematical process in Eqs. (33) and (34) is needed. The socalled Sobol indices or variance based sensitivity indices [18] are obtained. These indices express the share of variance of $Y$ that is due to a given input or inputs combination.

$$
S_{i}=\frac{D_{i}(Y)}{\operatorname{Var}(Y)}, S_{i j}=\frac{D_{i j}(Y)}{\operatorname{Var}(Y)}, \ldots
$$

There are $2^{d}-1$ indices. The total indices or total effects are introduced as follows:

$$
S_{(i)}^{t o t}=S_{i}+\sum_{j \neq i} S_{i j}+\sum_{i \neq j, j \neq k, j<k} S_{i j k}+\ldots=\sum_{l \in \# i} S_{l},
$$

where $\# i$ are all the subsets of $\{1,2, \ldots, d\}$ including $i$. In practice, when $d$ is large, only the main effects and the total effects are computed, thus giving a good information on the model sensitivities. The most informative situations are noted in Eqs. (37)-(38). Eq. (37) means the output is independent of $i$ th variable and Eq. (38) shows that the output is 
dependent of $i$ th variable only. The difference between these two values stated on interaction of one input parameters with other input parameters.

$$
\begin{aligned}
& S_{(i)}^{t o t}=S_{i}=0 ; \\
& S_{(i)}^{t o t}=S_{i}=1 .
\end{aligned}
$$

\section{Numerical results and discussions}

Numerical results for presented model is investigated for a case study. The specifications of AM-AFM cantilever are tabulated in Table 1 that are adopted from [17]. In each problem the excitation frequency of base is set as the resonance frequency of same problem. $w_{\text {set }}$ is set $60 \mathrm{~nm}$ and $Y_{0}$ is assumed $60 \mathrm{~nm}$. This large amount of base amplitude assures that the cantilver works in attractive and repulsive force regimes both [2]. Magnitude of nonlinear nanoscale tip sample interaction force is adopted from [24] for scanning condition.

Verification of model is checked with comparing with experimental and theoretical results in [17] that are tabulated in Table 2. The results of present model is tabulated in Table 3 for three different assumptions. Percent of error is defined as average of absolute deviation of each mode with respected to experimental data in the same mode.

Table 1

List of parameters of AM-AFM cantilever

\begin{tabular}{|l|l|l|l|}
\hline Parameter & Value & Description & Unit \\
\hline$L$ & 474 & Length & $\mu \mathrm{m}$ \\
\hline$b_{t}$ & 53 & Top Wide & $\mu \mathrm{m}$ \\
\hline$b_{b}$ & 50 & Bottom Wide & $\mu \mathrm{m}$ \\
\hline$t_{1}$ & 1.66 & Cantilever Tickness & $\mu \mathrm{m}$ \\
\hline$t_{2}$ & 30 & Coating Tickness & $\mathrm{nm}$ \\
\hline$E_{1}$ & 160 & Cantilever Elastic Mod. & $\mathrm{Gpa}$ \\
\hline$E_{2}$ & 68 & Coating Elastic Mod. & $\mathrm{Gpa}$ \\
\hline$v_{1}$ & 0.27 & Cantilever Poisson Ratio & - \\
\hline$v_{2}$ & 0.38 & Coating Poisson Ratio & - \\
\hline$k_{1}$ & 0.8484 & Cantilever Shear Factor & - \\
\hline$k_{2}$ & 0.8529 & Coating Shear Factor & - \\
\hline$\rho_{1}$ & 2329 & Cantilevr Specific Mass & $\mathrm{Kg} / \mathrm{m}^{3}$ \\
\hline$\rho_{2}$ & 2698.9 & Coating Specific Mass & $\mathrm{Kg} / \mathrm{m}^{3}$ \\
\hline$m_{t i p}$ & 0 & Tip mass & $\mathrm{Kg}$ \\
\hline$a_{a}$ & $-1 \times 10^{-29}$ & Attractive Amplitude term & - \\
\hline$a_{r}$ & $1 \times 10^{-86}$ & Repulsive Amplitude Term & - \\
\hline$\gamma_{a}$ & -2 & Attractive Power Term & - \\
\hline$\gamma_{r}$ & -8 & Repulsive Power Term & - \\
\hline & & & \\
\hline
\end{tabular}

It is seen that the present model can predict the experimental data in good accuracy and effect of backside coating is important even in the range of $30 \mathrm{~nm}$. As it is seen in Table 3 if the tip length assumed shorter but the effect of tip mass entered the error is decreased to lower range. To investigate the effects of backside coating different physical range of related parameters are studied. The minimum and maximum of parameters are tabulated in Table 4.
Table 2

Resonance frequency in $\mathrm{KHz}$ adopted from [17]

\begin{tabular}{|l|c|c|c|}
\hline Mode & Experiment & FEM & Theory \\
\hline$\# 1$ & 9.978 & 9.978 & 9.939 \\
\hline$\# 2$ & 62.620 & 62.265 & 62.286 \\
\hline$\# 3$ & 174.800 & 174.567 & 174.401 \\
\hline$\# 4$ & 343.000 & 342.354 & 341.110 \\
\hline$\# 5$ & 567.500 & 566.909 & 564.949 \\
\hline$\# 6$ & 848.000 & 848.053 & 843.936 \\
\hline Error(\%) & 0.000 & 0.304 & 0.677 \\
\hline
\end{tabular}

Table 3

Resonance frequency in $\mathrm{KHz}$ for present model

\begin{tabular}{|l|c|c|c|}
\hline \multirow{2}{*}{ Mode } & \multicolumn{3}{|c|}{ Present Model } \\
\cline { 2 - 4 } & $\begin{array}{c}L=474 \mu \mathrm{m} \\
\text { Uncoated }\end{array}$ & $\begin{array}{c}L=474 \mu \mathrm{m} \\
\text { Coated }\end{array}$ & $\begin{array}{c}L=470 \mu \mathrm{m} \\
\text { Coated }+M_{n}=1 \%\end{array}$ \\
\hline$\# 1$ & 9.886 & 9.996 & 9.973 \\
\hline$\# 2$ & 61.948 & 62.639 & 62.535 \\
\hline$\# 3$ & 173.442 & 175.375 & 175.177 \\
\hline$\# 4$ & 339.831 & 343.617 & 343.408 \\
\hline$\# 5$ & 561.667 & 567.920 & 567.859 \\
\hline$\# 6$ & 838.854 & 848.186 & 848.500 \\
\hline Error(\%) & 0.859 & 0.246 & 0.171 \\
\hline
\end{tabular}

Table 4

Physical rang of backside coating parameters

\begin{tabular}{|c|c|c|c|}
\hline Parameter & Minimum & Maximum & Unit \\
\hline$t_{2}$ & 0 & 500 & $\mathrm{~nm}$ \\
\hline$E_{2}$ & 0 & 700 & $\mathrm{Gpa}$ \\
\hline$\rho_{2}$ & 0 & 25000 & $\mathrm{Kg} / \mathrm{m}^{3}$ \\
\hline$v_{2}$ & 0 & 0.5 & - \\
\hline
\end{tabular}

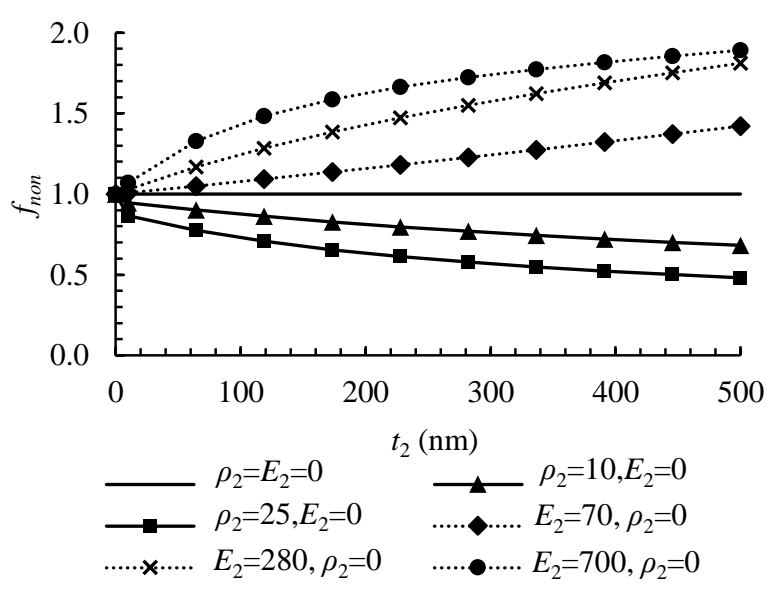

Fig. 3 Variation of $f_{\text {non }}$ with $t_{2}$ for inelastic and massless assumption of coating ( $E_{2}$ is in Gpa and $\rho_{2}$ in $\left.\mathrm{g} / \mathrm{cm}^{3}\right)$

$f_{n o n}$ is defined as the resonance frequency of each problem divided to resonance frequency of first mode of uncoated AM-AFM cantilever (here $9.8856 \mathrm{KHz}$ ). Two important assumption are the inelastic $\left(E_{2}=0\right)$ assumption and massless $\left(\rho_{2}=0\right)$ assumption for coating. These two assumptions are plotted in Fig. 1 for $f_{\text {non }}$. Fig. 3 demonstrates that these assumption are not valid for proper modelling of 
coating effects even for small thickness. The inelastic assumption is under estimated and the massless assumption is over estimated in prediction of $f_{\text {non }}$.

GSA based on Sobol method is applied to $f_{\text {non }}$ as output of dynamic system when the cantilever is faraway of the sample by coding in MATLAB. The Sobol indices and total indices of backside coating parameters are plot in Fig. 4. Results demonstrate that the effect of coating's Poisson Ratio is negligible in output and the most important factor is $\rho_{2}$, however the effect of $E_{2}$ is important too. Thus in next steps the effect of Poission ratio is neglected.

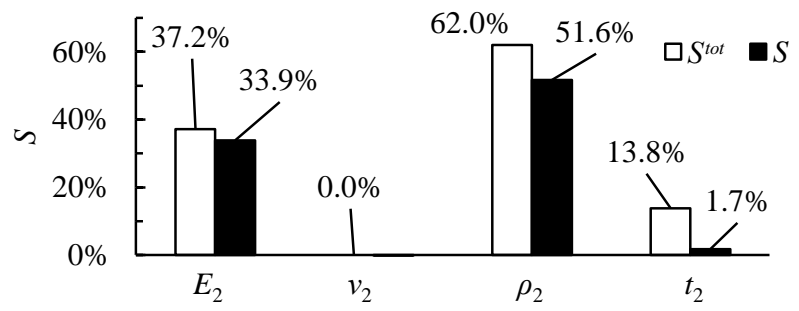

Fig. 4 Sensitivity indices for backside coating parameters for $f_{\text {non }}$ as output

To calculate the amplitude of response, time domain response of cantilever is determined by coding in MATLAB and amplitude of response in excitation frequency is calculated by Fast Fourier Transform (FFT) of time response. Sampling frequency of FFT is set such that the frequency resolution remains minimum in $10 \mathrm{~Hz}$ level. This process is applied to both scanning and faraway conditions of AM-AFM. The magnitude of amplitude in faraway condition and in scanning condition is defined $A_{0}$ and $A_{1}$ respectively for each problem. The ratio of $A_{1} / A_{0}$ is defined $A_{n o n}$ that is the monitoring signal in AM-AFM [2, 3].

Fig. 5 shows variation of $A_{n o n}$ while the thickness of backside coating is assumed 100nm. Different coating that are frequently used for AM-AFM are showed as a surface in this situation. It should be remarked that the value of $A_{n o n}$ is lower that unit for uncoated AM-AFM cantilever. This reduction is due to effect of nonlinear tip-sample interaction force. It is seen value of $A_{\text {non }}$ is decreased with coating effects. The amount of this reduction is significant when comparing it with the decrease of amplitude for uncoated cantilever. $A_{\text {non }}$ is waned generally with the increase of elastic modulus and specific mass.

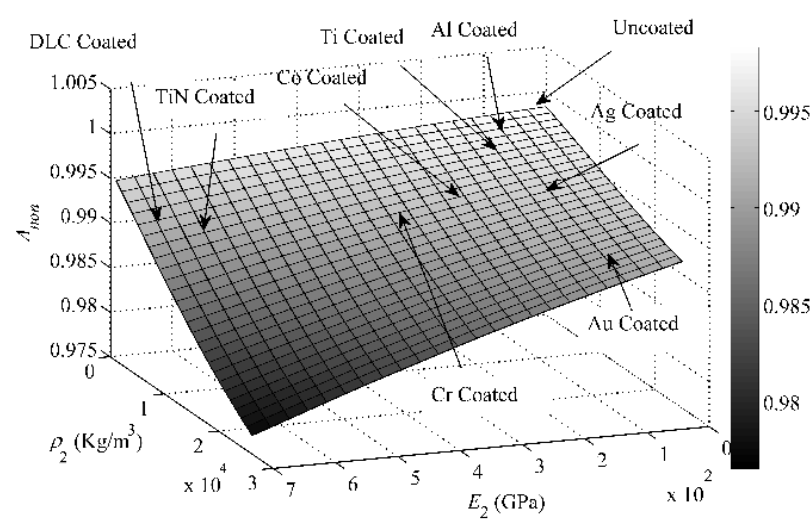

Fig. 5 Variation of $A_{n o n}$ in fixed magnitude of $t_{2}=100 \mathrm{~nm}$

In Fig. $6 E_{2}$ is assumed as the elastic modulus of chrome $\left(E_{C r}\right)$ and the effects of $t_{2}$ and $\rho_{2}$ on $A_{n o n}$ is figured.
It is seen with increasing the magnitude of both $t_{2}$ and $\rho_{2}$ the nonlinearity of monitoring signal is increased, however in low amount of $t_{2}$ (about $10 \mathrm{~nm}$ ) the changes in $A_{n o n}$ is inclined to linear. The trend is always decreasing.

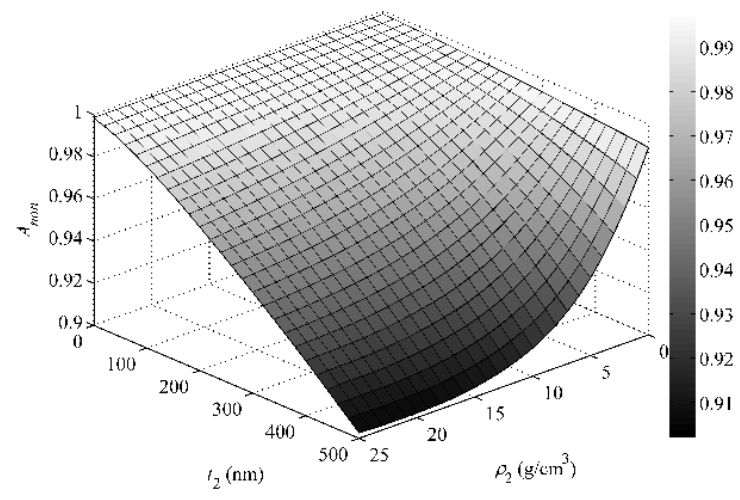

Fig. 6 Variation of $A_{\text {non }}$ in fixed magnitude of $E_{2}=E_{C r}$

In Fig. $7 \rho_{2}$ is assumed constant equal to the specific mass of Gold $\left(\rho_{2}=\rho_{A u}\right)$. Quantitatively changes in $A_{n o n}$ for different values of $E_{2}$ seems linear. Again, changes in $A_{\text {non }}$ are more linear in small value of $t_{2}$. Generally, the nonlinear trend is seen in Figs. 3-5.

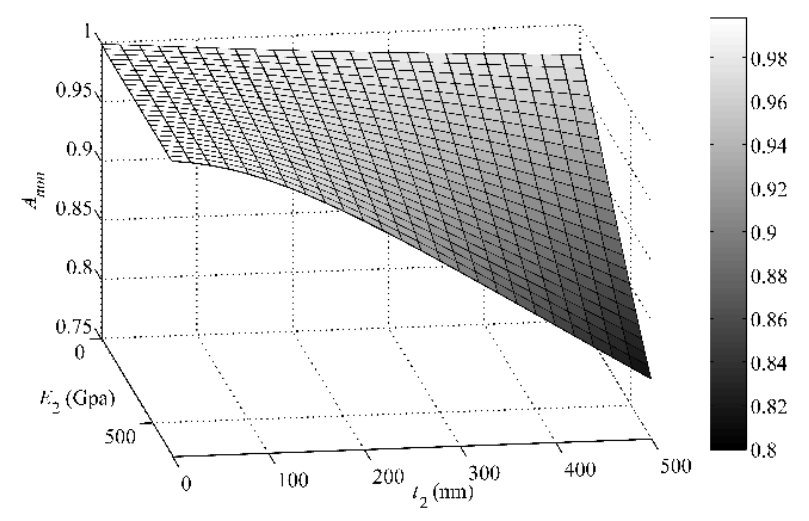

Fig. 7 Variation of $A_{n o n}$ in fixed magnitude of $\rho_{2}=\rho_{A u}$

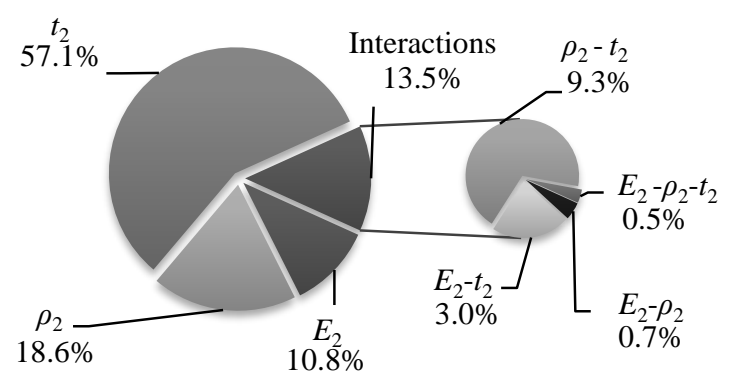

Fig. 8 Sobol indices of backside coating parameters for $A_{0}$ as output of dynamic system

Here the Sobol indices and total indices are determined for $A_{n o n}$ and $A_{0}$. This process is applied for these two different outputs for an important reason. Authors observed in their results that the effect of backside coating is different for $A_{0}$ and $A_{1}$. This different amplitude of response for faraway condition and scanning situation demonstrate that backside coating parameters affects the output signal of AMAFM.

Fig. 8 shows the Sobol indices for $A_{0}$ as output. In this case $t_{2}$ and $\rho_{2}$ and $E_{2}$ are the most influencing parameters 
for the model output variance according to first order index. The sum of first order indices of the parameters shows $86.5 \%$ which illustrate high interaction between studied parameters. Between the all interaction indices the share of thickness and density interaction is the most important and the third order interaction is negligible.

Fig. 9 shows the same indices for the monitoring signal of AM-AFM. The value of indices are different in all cases for $A_{n o n}$ but the sequence of most influence parameters are remained the same as for $A_{0}$. The changes in indices of $E_{2}$ and $\rho_{2}$ are neglectable. The variations are important in indices related to $t_{2}$ and interaction terms. The change is decreasing for $t_{2}$. Between all interaction indices the most change is related to thickness and specific mass interaction that is increasing. These changes demonstrate that the nonlinear nano-scale tip sample force affects the amount of $A_{\text {non }}$ due to backside coating and the dynamic response is different for scanning and faraway situations.

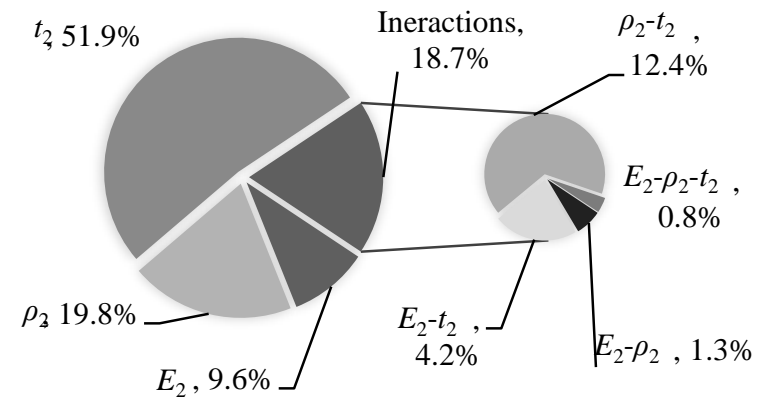

Fig. 9 Sobol indices of backside coating parameters for $A_{n o n}$ as output of dynamic system

The comparison of first order Sobol indices and total indices are plotted for both outputs in Fig. 10. The total sensitivity index in Fig. 10 shows that the $S^{\text {tot }}$ has a consistent trend as the $S$, that is, the ranking of parameters by first order values agreed well with those from $S^{\text {tot }}$ values. It is seen that all the $S^{\text {tot }}$ values are greater than first order indices. This again suggests each parameter interacts as a pair, or more with the other parameters that is discussed previously. The value of $S_{t o t}$ for both inputs have negligible changes for $t_{2}$. This is because of decreasing the first order and increasing of interactions related to $t_{2}$ that is caused for little change in total index. The most change for total index is seen for $\rho_{2}$.

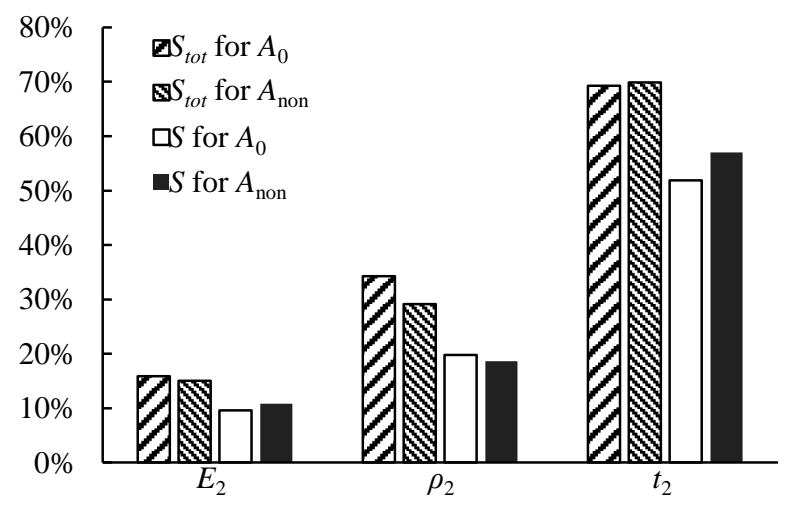

Fig. 10 Comparison of sensitivity indices for backside coating parameters for $A_{0}$ and $A_{\text {non }}$ as output

\section{Conclusions}

In this study, mathematical model of AM-AFM cantilever was derived based on Timoshenko beam theory with concentrated end mass. Effect of backside coating was included using extended Hamiltonian principle. The general form of nano-scale nonlinear tip-sample interaction force was involved as time variant boundary condition problem. The equations of motion were homogenized and combined to high order coupled PDEs of motion. The trapezoidal cross section was assumed for AM-AFM cantilever and all the calculations were applied for transformed cross section of area. Mode shapes of problem were derived for coated cantilever. The GSA based on Sobol's method was introduced which is able to predict the interaction of input parameters for dynamic nonlinear systems. In the faraway situation the natural frequency of coated cantilever was calculated for a case study and was validated with experimental results in literature. It was showed the error is lower for shorter cantilever with tip mass. The results were compared with previously introduced theoretical models too. Physical range of backside coating parameters were introduced. Two wellknown assumptions (inelastic and massless assumptions) were plotted. It was demonstrated that, these assumptions are not proper choice for modelling of coating effects in AM-AFM and the effect of backside coating should not neglect in mathematical modeling of AM-AFM. GSA on these parameters were applied to non-dimensional resonance frequency for faraway situation. It was showed the effect of Poisson ratio of coating is negligible. For a constant value of every coating parameters the variation of non-dimension amplitude in scanning condition was investigated. It is showed qualitatively that for small magnitude of coating thickness the behavior is inclined linear. First order and total indices of backside coating parameters for faraway and scanning situation of AM-AFM were determined and compared. It was showed the sequence of indices remains unchanged for both cases and the most important parameters are thickness, specific mass and elastic modulus of coating respectively. However, the value of indices are different for each condition. It was showed the interactions between parameters are important in variance of outputs and the related indices are large enough. Comparison between the first order indices and total indices were discussed. It was remarked difference between the GSA indices for faraway and scanning situation shows that the effect of coating is different in both condition.

\section{References}

1. Binnig, G.; Quate, C. F; Gerber, Ch. 1986 Atomic force microscope, Physical Review Letters 56: 930-933. http://dx.doi.org/10.1103/PhysRevLett.56.930.

2. Garcia, R. 2011. Amplitude Modulation Atomic Force Microscopy, Weinhein: WILEY-VCH Verlag GmbH \& Co. KGaA, 193 p. http://dx.doi.org/10.1002/9783527632183.

3. Shih, P.J. 2012. Tip-jump response of an amplitudemodulated atomic force microscope, Sensors 12: 66666684. http://dx.doi.org/10.3390/s120506666.

4. Marinello, F.; Passeri, D.; Savio, E. 2012. Acoustic Scanning Probe Microscopy, Berlin: Springer-Verlag Berlin Heidelberg, 494p. 
http://dx.doi.org/10.1007/978-3-642-27494-7.

5. Kahrobaiyan, M.H.; Rahaeifard, M.; Ahmadian, M.T. 2011. Nonlinear dynamic analysis of a $v$ shaped microcantilever of an atomic force microscope, Applied Mathematical Modelling 35: 5903-5919. http://dx.doi.org/10.1016/j.apm.2011.05.039.

6. McCarty, R.; Carmichael, B.; Mahmoodi, S.N. 2014. Dynamic analysis of tapping atomic force microscopy considering various boundary value problems, Sensors and Actuators A: Physical 216: 69-78. http://dx.doi.org/10.1016/j.sna.2014.04.036.

7. Sader, J.E.; Larson, I.; Mulvaney, P.; White, L.R. 1995. Method for the calibration of atomic force microscope cantilevers, Review of Scientific Instruments 66: 3789-3798.

http://dx.doi.org/10.1063/1.1145439.

8. Georgakaki, D.; Mitridis, S.; Sapalidis, A.A.; Mathioulakis, E.; Polatoglou, H.M. 2013. Calibration of tapping afm cantilevers and uncertainty estimation: comparison between different methods, Measurement 46: 4274-4281. http://dx.doi.org/10.1016/j.measurement.2013.08.010.

9. Salvadori, M.C.; Fritz, M.C.; Carraro, C.; Maboudian, R.; Monteiro, O.R.; Brown, I.G. 2001. Characterization of AFM cantilevers coated with diamond-like carbon, Diamond and Related Materials 10(12): 21902194. http://dx.doi.org/10.1016/S0925-9635(01)00502-7.

10. Salvadori, M.C.; Brown, I.G.; Vaz, A.R.; Melo, L.L.; Cattani, M. 2003. Measurement of the elastic modulus of nanostructured gold and platinum thin films, Physical Review B 67: 153404. http://dx.doi.org/10.1103/PhysRevB.67.153404.

11. Amadori, S. ;Campari, E.G.; Fiorini, A.L.; Montanari, R.; Pasquini, L.; Savini, L.; Bonetti, E. 2006. Automated resonant vibrating-reed analyzer apparatus for a non-destructive characterization of materials for industrial applications, Materials Science and Engineering: A, 442: 543-546.

http://dx.doi.org/10.1016/j.msea.2006.02.210.

12. Meng, L.; Huang, T., Wang, X.; Chen, Sh.; Yang, Zh.; Ren, B. 2015. Gold-coated AFM tips for tip-enhanced Raman spectroscopy: theoretical calculation and experimental demonstration, Optics Express 23: 1380413813. http://dx.doi.org/10.1364/OE.23.013804.

13. Schumacher, Z.; Miyahara1, Y.; Aeschimann, L.; Grütter, P. 2015. Improved atomic force microscopy cantilever performance by partial reflective coating, Beilstein Journal Nanotechnology 6: 1450-1456. http://dx.doi.org/10.3762/bjnano.6.150.

14. Allen, M.S.; Sumali, H.; Penegor, P.C. 2009. DMCMN: experimental/analytical evaluation of the effect of tip mass on atomic force microscope cantilever calibration, Journal of Dynamic Systems, Measurement and Control 131: 064501-10. http://dx.doi.org/10.1115/1.4000160.

15. Heim, L.; Rodrigues, T.S.; Bonaccurso, E. 2014. Direct thermal noise calibration of colloidal probe cantilevers, Colloids and Surfaces A: Physicochemical and Engineering Aspects 443: 377-383. http://dx.doi.org/10.1016/j.colsurfa.2013.11.018.
16. Song, Y.; Wu, S.; Xu, L.; Fu, X. 2015. Accurate calibration and uncertainty estimation of the normal spring constant of various AFM cantilevers, Sensors 15: 58655883. http://dx.doi.org/10.3390/s150305865.

17. Mendel, D.A.; Lowe, M.; Cuenat, A.; Cain, M.G.; Vallejo, E.; Ellis, D.; Mendels, F. 2006. Dynamic properties of AFM cantilevers and the calibration of their spring constants, Journal of Micromechanics and Microengineering 16: 1720-1733.

http://dx.doi.org/10.1088/0960-1317/16/8/037.

18. Sobol, I.M. 2001. Global sensitivity indices for nonlinear mathematical models and their Monte Carlo estimates, Mathematics and Computers in Simulation 55: 271-280. http://dx.doi.org/10.1016/S0378-4754(00)00270-6.

19. Korayem, M.H.; Taheri, M.; Ghasemi, M.; Badkoobehhezavh, H. 2015. Investigating the effective parameters in the Atomic Force Microscope-based dynamic manipulation of rough micro/nanoparticles by using the Sobol sensitivity analysis method, Simulation 91: 1068-1080. http://dx.doi.org/10.1177/0037549715615216

20. Omidi, E.; Korayem, A.H.; Korayem, M.H. 2013. Sensitivity analysis of nanoparticles pushing manipulation by AFM in a robust controlled process, Precision Engineering 37: 658-670. http://dx.doi.org/10.1016/j.precisioneng.2013.01.011.

21. Ghaderi, R. 2015. Dynamic modeling and vibration analysis of piezoelectric microcantilever in AFM application, International Journal of Mechanics and Materials in Design 11: 1-13. http://dx.doi.org/10.1007/s10999-015-9309-y.

22. Korayem, M.H.; Ebrahimi, N.; Moloodi S. 2012. Sensitivity analysis of carbon nanotube characteristics on dynamic behavior of tapping-mode atomic force microscopy, Nanoscience and Nanotechnology 2: 57-65. http://dx.doi.org/10.5923/j.nn.20120203.03.

23. Korayem, M.H.; Ghaderi, R. 2014. Sensitivity analysis of nonlinear vibration of AFM piezoelectric microcantilever in liquid, International Journal of Mechanics and Materials in Design 10: 121-131. http://dx.doi.org/10.1007/s10999-013-9235-9.

24. Tetard, L.; Passian, A.; Thundat, T. 2009. New modes for subsurface atomic force microscopy through nanomechanical coupling, Nature Nanotechnology 5: 105109. http://dx.doi.org/ 10.1038/nnano.2009.454.

25. Rao, S.S. 2007. Vibration of Continuous Systems, Hoboken: John Wiley \& Sons Inc., 720p. http://dx.doi.org/10.1002/9780470117866.

26. Saltelli, A.; Ratto, M.; Andres, T.; Campolongo, F.; Cariboni, J.; Gatelli, D.; Salsana, M.; Tarantola, S. 2008. Global Sensitivity Analysis - The Primer, Chichester: John Wiley \& Sons Inc., 304p. http://dx.doi.org/10.1002/9780470725184.

27. Grant, D.A. 1978. The effect of rotary inertia and shear deformation on the frequency and normal mode equations of uniform beams carrying a concentrated mass, Journal of Sound and Vibration 57: 357-365. http://dx.doi.org/10.1016/0022-460X(78)90316-4.

28. Esmailzadeh, E.; Jalili, N. 1998. Parametric Response of Cantilever Timoshenko beams with tip mass under 
harmonic motion, International Journal of Non-linear Mechanics 33: 765-781.

http://dx.doi.org/10.1016/S0020-7462(97)00049-8.

29. Hoeffding, W. 1948. A class of statistics with asymptotically normal distributions, The Annals of Mathematical Statistics 19: 293-325.

http://dx.doi.org/10.1214/aoms/1177730196.

Kaveh E. Torkanpouri, Hassan Zohoor, Moharam H. Korayem

\section{GLOBAL SENSITIVITY ANALYSIS OF BACKSIDE COATING PARAMETERS ON DYNAMIC RESPONSE OF AM-AFM}

\section{S u m m a r y}

Influence of backside coating parameters on dynamic response of AM-AFM is modeled. Timoshenko based beam model is used and the general nonlinear interaction force is enclosed. The coupled ODEs of motion are achieved. The mode shapes are determined for coated cantilever and the natural frequency of each mode is validated with experimental results. It is showed a shorter cantilever with tip mass have better accuracy and inelastic and massless assumptions are not proper choice for modelling of the coating. The assumed mode method is used to determine time response of system for faraway and scanning condition of AM-AFM. Amplitude of response is calculated by FFT of time domain. Sensitivity indices of backside coating parameters are determined based on Sobol method in their physical range. It is showed the sequence of indices remains unchanged. The most important parameters and interactions on output variance are introduced. The results are concluded.

Keywords: AM-AFM, Global sensitivity analysis, Nonlinear vibration, Coated cantilever, Backside Coating parameters, Timoshenko beam theory

Received January 01, 2016

Accepted April 14, 2017 